\title{
Genetic variants of the oppA gene are involved in metabolic regulation of surfactin in Bacillus subtilis
}

\author{
Xiaoyu Wang $\mathbb{D}$, Zhiyi Chen, Hui Feng, Xi Chen and Lihui Wei
}

\begin{abstract}
Background: Bacillus subtilis 916 has been identified as an effective biocontrol agent against Rhizoctonia solani, the causal pathogen of rice sheath blight, under greenhouse and field conditions. HPLC analysis showed that surfactin, a member of the lipopeptide family produced by B. subtilis, was the major antimicrobial substance.

Results: Previously, we obtained a mutant strain of B. subtilis 916, Bs-H74, which produced significantly more surfactin than the wild type and presented $10 \%$ stronger inhibitory activity against $R$. solani. To explore the molecular mechanism underlying the higher surfactin productivity in the mutant, high-throughput proteomic analysis was carried out to analyze the differential protein expression. Our results showed that several differentially expressed proteins are involved in OppA, DegU and Carbon Catabolite Repression (CCR) regulatory pathways, which could be positively or negatively associated with surfactin biosynthesis. At both transcriptional and translational levels, we suggested that OppA may play a key role in surfactin synthesis regulation. Based on the above findings, we proposed the hypothesis that a point mutation in the oppA gene may lead to changes in oligopeptides acquisition in B. subtilis, and then the changed oligopeptides may activate or suppress the global regulatory protein, CcPA in the CCR pathway, and ComA and DegU may indirectly regulate surfactin synthesis in Bs-H74. To further explore the regulatory mechanisms in Bs-H74, metabolomics analysis was performed in this study. Interestingly, only 16 metabolites showed changes in abundance in Bs-H74 compared to Bs-916. Neohesperidin, a type of natural flavanone glycosides from citrus with a range of biological activities, increased by 18 times over the wild type Bs-916. This result implied exciting findings in regulatory mechanisms by OppA protein.
\end{abstract}

Conclusions: In summary, this study has revealed the mechanisms underlying the improved antagonistic property with increased surfactin production in Bs-H74 at the gene, protein and metabolic levels, which may help to comprehend the map of the regulatory networks in B. subtilis. Findings from our work have provided a solid physical and theoretical basis for practically applying metabolic and genetic engineering to achieve improved and high-yielding biocontrol strains.

Keywords: Surfactin, oppA, Metabolomics, CCR, сcpA, Global regulator

\section{Background}

It is essential to control plant diseases for the abundance of foods, feeds and fibers for keeping up quickly growing global population. Agriculturists have been primarily dependent upon pesticides and fertilizers for maximal agricultural production. However, during recent years,

*Correspondence: weilihui@jaas.ac.cn

Institute of Plant Protection, Jiangsu Academy of Agricultural Sciences, Nanjing 210014, China people have changed their attitude towards pesticides and fertilizer due to the rising risks of ecological pollution, and the negative impact of pesticide residuals on human health and the earth ecosystem. Researchers have occupied their consideration towards the alternative of natural antagonistic microorganisms to control plant diseases. Bacillus species have abilities to duplicate quickly, become adaptive to different environmental conditions, and they also possess antagonism against a wide range of 
phytopathogenic microorganisms. Bacillus species produced volatile compounds to promote plant growth and activate plant defense by triggering the induced systemic resistance. Kloepper et al. [1] investigated the elicitation effect of different Bacillus species, such as Bacillus subtilis, B. pumilus, B. cereus, B. pasteurii, B. amyloliquefaciens, B. mycoides, and B. sphaericus on resistance induction in vegetable, Arabidopsis sp., loblolly pine and tobacco under field conditions and green house. Significant reduction in disease incidence and severity was observed in various diseases caused by bacteria, fungi, viruses, oomycetes and nematodes.

Bacillus subtilis strains produce a wind range of bioactive compounds that have awesome possibilities for horticultural and biocontrol applications. One in-depth study class is comprised of the peptide antibiotics and amphiphilic biosurfactants. Common examples from this class include surfactin [2-4], iturin compounds [5], and fengycin [6]. Surfactin is an extracellular peptide antibiotic, and its production in B. subtilis has been reported. Surfactin synthesis required the expression of $s f p$ gene [7]. The $s r f A$ locus, an operon spanning over $25 \mathrm{~kb}$, is required for the production of surfactin [8]. SrfA expression is induced at the end of exponential growth and is regulated by the products of two-component regulatory genes comP and comA and the srfA promoter region (PsrfA) [9-13].

Because of the low yield of surfactin in B. subtilis strains, commercial production of surfactin has not been established. Attempts in optimizing the fermentation process have been made in recent studies to improve surfactin production [14-17]; however, these efforts failed to realize a commercially viable and profitable level of surfactin production. Some researchers have been focusing on screening for surfacing overproducing mutants or establishing genetically modified $B$. subtilis strains [18]. For example, recombinant strains with a modified promoter region of the $s r f A$ operon were constructed to induce $B$. subtilis to produce surfactin constitutively [19]; The $y n g H$ gene was overexpressed in B. subtilis, the surfactin production increased to $13.37 \mathrm{~g} / \mathrm{L}$ [20]; A series of engineered strains with the modularization of metabolic pathways were constructed, the highest surfactin titer increased to $12.8 \mathrm{~g} / \mathrm{L}$ [21]; Through the successful expression of Vitreoscilla hemoglobin gene, surfactin titer increased to $10.2 \mathrm{~g} / \mathrm{L}$ [22]. Unfortunately, these measures had achieved limited success due to the complex intrinsic regulatory network underlying $\operatorname{srfA}$ expression $[18,19]$. So far it remains difficult to produce surfactin in large quantities economically.

The oligopeptide transport system Opp is the significant and conserved transporter utilized by the Bacillus species and other microorganisms to import peptides.
In the B. subtilis, Opp operon consists of five subunitsOppA, OppB, OppC, OppD, and OppF (Additional file 1: Figure S1). OppA protein plays an important role in the initial recognition and binding of substrates [23-26]. In addition to the combination of oligopeptides to provide amino acid nutrition to cells, some of OppA also play a role in other cellular activities, such as signal transduction, cell wall peptide metabolism, and as a molecular chaperone to assist in proteins fold in periplasmic space. Adrianne et al. reported that mutation of opp and app in Clostridium difficile seriously affected the expression of earlier sporulation genes and increased the amounts of spores. And opp and app mutants showed greater virulence in the hamster [27]. After OppA binds to the substrate, how to coordinate with other subunits to transport the substrate into the cell has not been reported in detail. So far, to the best of our knowledge, there is no report on the regulatory effect of Opp system on surfactin production.

Carbon Catabolite Repression (CCR) is a regulatory mechanism. CcpA is known as a central regulator of CCR in B. subtilis. After inactivation of $c c p A$ gene, it can release the inhibition of $\alpha$-amylase synthesis gene amyE by glucose [28-33]. CcpA binds to the catabolite-responsive element ( $\mathrm{cre}$ ) located in the transcription start region of $a m y E$, does not require the cofactor Hpr. However, subsequent studies have shown that in most cases the combination of CcpA and cre sites requires cofactor protein P-(Ser)-HPr. The activity of P-(Ser)-HPr is affected by the ratio of intracellular ATP to inorganic phosphate (ATP/Pi), the intracellular concentration of 1,6-diphosphate fructose (FBP) and 6-phosphate glucose (G6P) [34, 35]. Many key enzyme genes in the glycolytic pathway are regulated by CcpA. Previous report have shown that activation of the gap operon is dependent on the presence of CcpA [30]. CcpA can directly or indirectly inhibit the expression of the citrate synthase gene citZ involved in the TCA cycle [36, 37]. In Streptococcus gordonii, CcpA influence the expression of adhesin genes, competence development and biofilm formation [27]. So far, the networks between CcpA and surfactin production has not been established.

Bacillus subtilis 916 was identified in a screening for biocontrol agent against the rice sheath blight caused by Rhizoctonia solani. It demonstrated its effectiveness in promoting plant growth and suppressing plant pathogenic organisms [38] in greenhouse and field tests. Previously, we obtained a mutant strain, Bs-H74, by means of low energy ion implantation in wild-type Bs-916 [39], which produces a significantly higher amount of surfactin and has better effects on controlling $R$. solani. High performance liquid chromatography (HPLC) analysis indicated that the major antagonistic substance produced by Bs-916 
and Bs-H74 is surfactin, a member of the lipopeptide family [39]. In order to decipher the mechanisms underlying the higher productivity of surfactin in Bs-H74, in this study, we compared the sequences of PsrfA and $\operatorname{comA}$ in Bs-H74 and the wild type Bs-916, and found that the sequences were identical. We further performed genomic, proteomic and metabolic analysis to explore new clues to the improved level of surfactin in Bs-H74. Results from this study may lead to a better understanding of the regulation of surfactin production, and therefore expedite the industrial production of surfactin and agricultural utilization of Bacillus spp. as biocontrol agents.

\section{Results and discussion}

\section{ComA gene and srfA promoter sequence analysis}

The $\operatorname{comA}$ gene and $\operatorname{SrfA}$ promoter (PsrfA) were amplified from Bs-H74 and Bs-916 using primers listed in Table 4. PsrfA and $\operatorname{com} A$ sequences were aligned by the clustW program. Our data showed that the PsrfA and $\operatorname{com} A$ sequences were $100 \%$ conserved between the Bs-H74 and Bs-916 strains (data not shown). These results suggested that promotion in surfactin production in $\mathrm{Bs}-\mathrm{H} 74$ may be due to other regulatory mechanisms.

\section{Identification of differentially expressed proteins}

We compared the 2-DE profiles of soluble proteins from Bs-916 and Bs-H74 mutant strains and found 183 protein spots differed between the strains by twofold or more. 74 protein spots were identified by MS analysis and their complete peptide fingerprints were obtained. After excluding unknown or non-Bacillus proteins, 32 proteins were found to have decreased expression, and 15 proteins were found to have increased expression in the Bs-H74 mutant compared to the wild type Bs-916. BLAST searches against the NCBI $\mathrm{nr}$ database with Mascot revealed that these differentially expressed proteins may function in nucleosides, carbohydrates, and amino acids modification (Table 1). CitB, GapA RpoA, AcoL, PurA, FtsZ, PupG and GlnA protein were found to overabundant in the Bs-H74; FbaA, HutI and IolD protein were found to have decreased expression. As above 11 proteins were functionally annotated to CcpA regulation. PutC, IspA, RocA were found to overabundant in the Bs-H74; RocD proteins were found to have decreased expression. 4 proteins were functionally annotated to CodY regulation. OppA protein found to be overabundant were functionally annotated to $\mathrm{ABC}$ transporters.

\section{Functional annotation of proteins}

The overrepresentation of GO categories among the 47 proteins showing a relative change in abundance in Bs-H74 mutant was assessed using BiNGO. The Bs-H74 mutant has modulated the abundance of proteins functionally related to transcription, translation and various metabolic processes, such as small molecules formation and RNA processing (Table 1). Protein functional annotation against KEGG Orthology (KO) showed that several proteins were overabundant in the Bs- $\mathrm{H} 74$ mutant in comparison to the wild type. According to the proteome data and reference, these proteins were categorized into 3 subgroups: (i) carbon catabolite repressors and their regulators, (ii) $\mathrm{ABC}$ transporters, (iii) transcription factors and their regulators.

Expression levels of several proteins involved in Carbon Catabolite Repression (CCR) aroused our attention. GapA, glyceraldehyde 3-phosphate dehydrogenase, expressed more than threefold in Bs-H74. RT-PCR was used to determine the transcription levels of $c g g R, c c p A$ and gapA in both the wild type Bs-916 and the mutant Bs-H74 strains. The five genes, $c g g R, c c p A, f b p$, citB and gapA were upregulated in the H74 strain (Fig. 1). AmyE gene was downregulated, cit $Z$ gene was minor upregulated. $F b p, c g g R$ and $a m y E$ gene expression were consistent with previous reported result. But gapA, citZ and $c i t B$ gene expression were not consistent with previous reported result [24, 30, 32, 33]. Our explanation was that oppA gene not only regulate the expression of CCR-associated genes, but also regulate the expression of GRP, global regulator protein, for example, $\operatorname{com} A$ gene in Bs-H74. We suggested that regulatory mechanisms of OppA protein is complicated, further study may be carried out to elucidate the mechanisms.

Another upregulated protein is the OppA. The expression level of OppA increased by more than twofold in Bs-H74. Real-time-PCR further identify that transcription levels of $o p p A$ is upregulated. And $o p p F$, belonging to the Opp operon, as we predicted, is also upregulated (Fig. 2). Transcriptional regulatory protein DegU is a member of the two-component regulatory system DegS/ DegU, which plays an important role in the transition growth phase. Expression level of DegU increased by more than sevenfold in Bs-H74.

\section{Resequencing and mutations detection}

In this study, we found 8 non-synonymous mutations resulting from SNPs, deletions, insertions, and shifts in Bs-H74. Six of the non-synonymous mutations led to mutated genes that coded for OppA/AppA oligopeptide transport protein family, transcriptional repressor of sporulation, collagen-like protein, proline permease, and glycerol-L-phosphate dehydrogenase. Specifically, in this study, all SNPs resulted in amino acid substitutions. As we expected, one substitution, Trp for Arg, occurred in the OppA protein (Table 2). This mutation may be responsible for the up-regulated expression of OppA in Bs-H74. 
Table 1 Identification of differentially regulated cellular proteins of Bacillus subtillis

\begin{tabular}{|c|c|c|c|c|c|}
\hline Accession number & Gene & Description & $P$ value & Fold change & Log.Fold change \\
\hline 16078991 & DhaS & Aldehyde dehydrogenase & 0.00 & 0.05 & -4.41 \\
\hline 154687531 & GapA & Glyceraldehyde-3-phosphate dehydrogenase, ссрA indirectly regulated & 0.00 & 0.08 & -3.62 \\
\hline 154687186 & PckA & Phosphoenolpyruvate carboxykinase, $c c p N$ repression & 0.01 & 0.08 & -3.68 \\
\hline 15616502 & PutC & 1-Pyrroline-5-carboxylate dehydrogenase, codY repression & 0.00 & 0.10 & -3.31 \\
\hline 16078863 & CitB & Aconitate hydratase, ссрA regulated & 0.00 & 0.12 & -3.11 \\
\hline 52082083 & DegU & Two-component regulator, ссрA regulated & 0.01 & 0.09 & -3.50 \\
\hline 34763277 & RpoA & RNA polymerase alpha subunit, interact with the CcpA protein [47] & 0.00 & 0.13 & -2.97 \\
\hline 52080062 & AcoL & Dihydrolipoamide dehydrogenase, $с с p A$ regulated & 0.02 & 0.10 & -3.39 \\
\hline 16080734 & AtpB & ATP synthase & 0.02 & 0.14 & -2.83 \\
\hline 154685773 & MtnD & Acireductone dioxygenase, methionine salvage pathway & 0.00 & 0.14 & -2.83 \\
\hline 154684814 & ProA & 1-Pyrroline-5-carboxylate dehydrogenase, amino acid/nitrogen metabolism & 0.00 & 0.14 & -2.83 \\
\hline 154687206 & PurC & Naphthoate synthase, nucleotide metabolism & 0.05 & 0.11 & -3.21 \\
\hline 154686652 & PepT & Peptidase T (tripeptidase), utilization of peptides & 0.00 & 0.19 & -2.43 \\
\hline 37781613 & SodA & $\begin{array}{l}\text { SOD, important for survival of ethanol and paraquat stresses and at low } \\
\text { temperatures }\end{array}$ & 0.00 & 0.20 & -2.31 \\
\hline 154687051 & Pyk & Pyruvate kinase, glycolysis pathway & 0.01 & 0.19 & -2.38 \\
\hline 154687044 & Mdh & Malate dehydrogenase, ссрA regulated & 0.01 & 0.18 & -2.47 \\
\hline 154686893 & CymR & Pleiotropic regulator of sulfur metabolism, sulfur metabolism & 0.02 & 0.18 & -2.48 \\
\hline 154688140 & PurA & Adenylosuccinate synthetase, interact with the CcpA protein [47] & 0.01 & 0.24 & -2.06 \\
\hline 154684785 & YceD & Stress adaption protein & 0.00 & 0.24 & -2.05 \\
\hline 154685945 & FtsZ & Cell division protein, interact with the CcpA protein [47] & 0.09 & 0.16 & -2.63 \\
\hline 63146095 & IspA & Intracellular serine protease, codY repression & 0.06 & 0.16 & -2.65 \\
\hline 154686374 & PupG & Purine nucleoside phosphorylase, $с c p A$ regulated & 0.01 & 0.24 & -2.07 \\
\hline 194014533 & PdhB & Pyruvate dehydrogenase, TCA cycle & 0.00 & 0.26 & -1.96 \\
\hline 154687585 & ClpP & ATP-dependent Clp protease proteolytic subunit & 0.00 & 0.24 & -2.04 \\
\hline 154685670 & Ndh & NADH dehydrogenase (menaquinone 7 and no proton), respiration & 0.00 & 0.27 & -1.89 \\
\hline 154687897 & RocA & 1-Pyrroline-5-carboxylate dehydrogenase, codY repression & 0.05 & 0.22 & -2.17 \\
\hline 16080001 & Tpx & Putative peroxiredoxin, spx regulated & 0.00 & 0.25 & -2.03 \\
\hline 154688008 & РepT & Peptidase T, amino acid/nitrogen metabolism & 0.00 & 0.30 & -1.74 \\
\hline 154687826 & YwjH & Putative translaldolase, pentose phosphate pathway & 0.01 & 0.32 & -1.63 \\
\hline 216396 & $G \ln A$ & Glutamine synthetase, interact with the CcpA protein [47] & 0.05 & 0.20 & -2.35 \\
\hline 154687185 & MetK & S-Adenosylmethionine synthetase, methionine salvage pathway & 0.08 & 0.21 & -2.22 \\
\hline 16078208 & OppA & Oligopeptide ABC transporter, uptake of peptides/codY repression & 0.01 & 0.24 & -2.05 \\
\hline 221311046 & GbsR & Regulation of osmoprotection & 0.14 & 5.27 & 2.40 \\
\hline 154685807 & Dnak & Chaperone protein & 0.00 & 3.27 & 1.71 \\
\hline 154687392 & FadA & Acyl-CoA acetyltransferase, fatty acid degradation & 0.00 & 4.71 & 2.24 \\
\hline 52081769 & FadE & Acyl-CoA dehydrogenase, fatty acid degradation & 0.01 & 5.44 & 2.44 \\
\hline 154686777 & $\mathrm{IspH}$ & Non-mevalonate pathway, biosynthesis of lipids & 0.04 & 5.32 & 2.41 \\
\hline 154688074 & lolD & Formation of 5-deoxy-D-glucuronic acid, $с c p A$ regulated & 0.04 & 5.50 & 2.46 \\
\hline 154684631 & TufA & Elongation factor Tu & 0.01 & 5.42 & 2.44 \\
\hline 154688042 & Hutl & Imidazolonepropionase, $с с p A$ regulated & 0.00 & 4.47 & 2.16 \\
\hline 154688124 & RocD & Omithine-oxo-acid transaminase, codY repression & 0.01 & 4.74 & 2.24 \\
\hline 154685878 & PdhD & Dihydrolipoamide dehydrogenase, TCA cycle & 0.01 & 6.07 & 2.60 \\
\hline 154687798 & AtpA & ATP synthase subunit & 0.04 & 10.98 & 3.46 \\
\hline 154687827 & $\mathrm{FbaA}$ & Fructose-bisphosphate aldolase, interact with the CcpA protein [47] & 0.00 & 9.73 & 3.28 \\
\hline 1389732 & MetE & Methionine synthetase, methionine salvage pathway & 0.12 & 20.94 & 4.39 \\
\hline 154686979 & $\operatorname{Sdh} B$ & Succinate dehydrogenase, TCA cycle & 0.07 & 26.59 & 4.73 \\
\hline 154685475 & YhfE & Putative endoglucanase & 0.02 & 35.31 & 5.14 \\
\hline
\end{tabular}




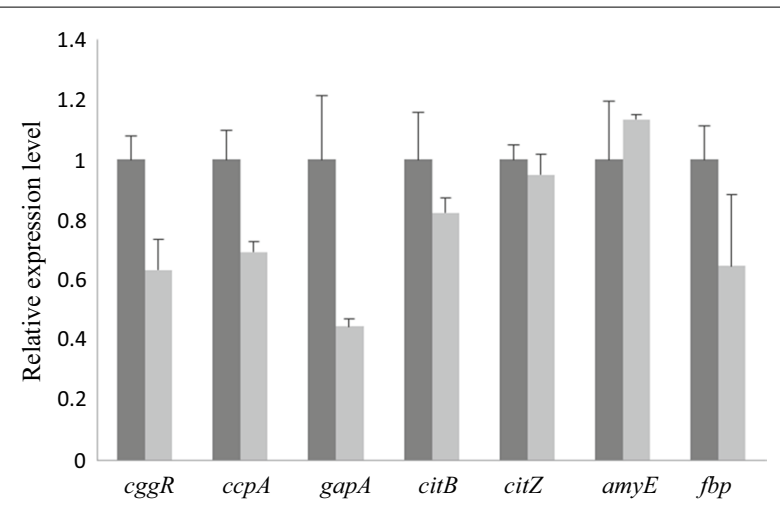

Fig. 1 Expression of CCR-associated genes in Bs-H74 and Bs-916. Grey column indicated wild type Bs-916 strain; black column indicated Bs-H74 strain

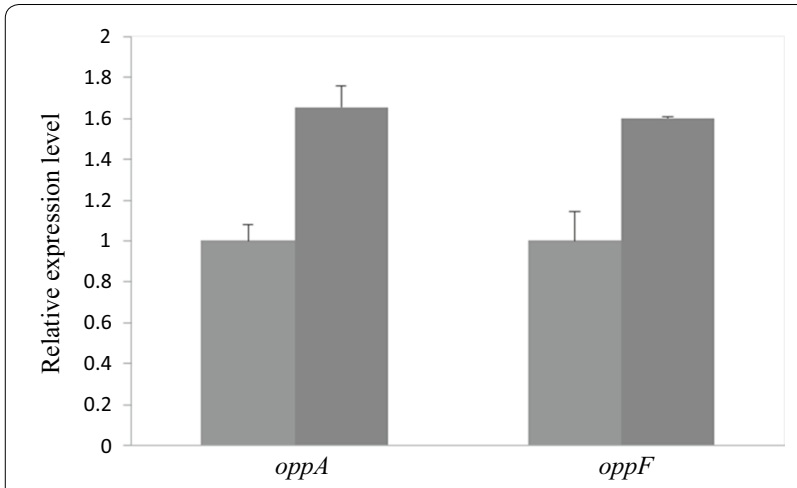

Fig. 2 Expression of opp system genes in Bs-H74 and Bs-916. Grey column indicated wild type Bs-916 strain; black column indicated Bs-H74 strain

\section{Metabolite identification}

In this study, metabolomics analysis resulted in the detection of 16 metabolites that showed changes in abundance in Bs-H74 compared to the wild type (Table 3). Neohesperidin, benzoylformic acid, oxamic acid, glucoheptonic acid, N6-acetyl-L-lysine, 4-hydroxyphenylacetic acid, 5'-methylthioadenosine, phenylpyruvate, 2-hydroxy-3-isopropylbutanedioic acid, and glycolic acid were found to be increased in abundance in Bs-H74. 2,4,6-trihydroxybenzophenone, conduritol b epoxide, 5-methoxytryptamine, lactulose, and monopalmitin were found to be decreased in abundance in Bs-H74. These metabolites are involved in amino acids metabolism, biosynthesis of secondary metabolites, biosynthesis of antibiotics, biosynthesis of amino acids, lipids and lipid-like molecules metabolism, and carbohydrates metabolic pathways, respectively.

Neohesperidin was found to have increased by 18 times in abundance in Bs-H74 compared to Bs-916 (Table 3). Previous research showed that Neohesperidin is a type of natural flavanone glycosides from citrus with a range of biological activities. It was first discovered in plants and usually distributed in wild orange, citrus aurantium, immature grapefruit. Neohesperidin activated AMPactivated protein kinase (AMPK) by phosphorylation and increased glucose consumption in HepG2 cells. These results indicated that Neohesperidin has a potential diabetes prevention and treatment effect [40]. Neohesperidin has also antibacterial, anti-allergic and anti-tumor pharmacological activities [41-43].

Glucoheptonic acid was found to have increased by two times in Bs-H74 than Bs-916. Glucuronic acid is a uronic acid in which C-6 hydroxyl group of glucose is oxidized to a carboxyl group. D-glucuronic acid generally is present as stable 3,6-lactone of the furan ring. D-glucopyranuronic acid is present in the oligosaccharide at the junction of the glycosaminoglycan chain, heparin and chondroitin.

The content of $5^{\prime}$-methylthioadenosine (MTA) was found to be increased in Bs-H74. MTA is a metabolite in the metabolism of polyamines and methionine. The molecular structure was reported in 1924. Precursor is $S$-adenosylmethionine. MTA promotes apoptosis of liver cancer cells and inhibits apoptosis of normal liver cells.

The content of N6-acetyl-L-lysine and glycolic acid increased in Bs-H74. Glycolic acid is the first member of

Table 2 Validated mutations in Bs-H74

\begin{tabular}{|c|c|c|}
\hline Gene & Mutation & Gene function \\
\hline RBAM_007770 & $\begin{array}{l}\text { V209A }(\text { GTA } \rightarrow \text { GCA); V230L } \\
\quad(\text { GTA } \rightarrow \text { TTA); I239V }(\text { ATA } \rightarrow \text { GTA })\end{array}$ & Collagen-like protein, colony and biofilm formation \\
\hline oppA & W519R $($ TGG $\rightarrow$ CGG) & Oligopeptide transport protein \\
\hline $\operatorname{rapA}$ & P259L (CCG $\rightarrow$ CTG) & Transcriptional repressor of sporulation, control of sporulation initiation \\
\hline $\operatorname{aram}$ & $\mathrm{Q} 226 \mathrm{~K}(\mathrm{CAG} \rightarrow \mathrm{AAG})$ & Glycerol-1-phosphate dehydrogenase, biosynthesis of phosphoglycerolipids \\
\hline RBAM_005790 & $C \rightarrow T$ intergenic $(+390 /-228)$ & Putative transcriptional repressor of sporulation and degradative enzymes production \\
\hline$C Z C D$ & + 38 bp intergenic $(-6 /-187)$ & Cation efflux, resistance against $\mathrm{Zn}, \mathrm{Cu}, \mathrm{Co}, \mathrm{Ni}$ \\
\hline ycgO & $1238 \mathrm{~N}(\mathrm{ATC} \rightarrow \mathrm{AAC})$ & High affinity proline permease, proline uptake \\
\hline nasD & $\mathrm{E} 307 \mathrm{D}(\mathrm{GAA} \rightarrow \mathrm{GAC})$ & Utilization of nitrite as nitrogen source \\
\hline
\end{tabular}


Table 3 Metabolites showing changes in abundance in Bs-H74 compared to Bs-916

\begin{tabular}{|c|c|c|c|c|}
\hline Metabolite & KEGG name & Formula & Fold & KEGG \\
\hline 2,4,6-Trihydroxybenzophenone & 2,4,6-Trihydroxybenzophenone & $\mathrm{C} 13 \mathrm{H} 10 \mathrm{O} 4$ & -1.06 & C06356 \\
\hline Conduritol b epoxide 2 & Conduritol b epoxide 2 & ND & -0.71 & ND \\
\hline 5-Methoxytryptamine 1 & 5-Methoxytryptamine & $\mathrm{C} 11 \mathrm{H} 14 \mathrm{~N} 2 \mathrm{O}$ & -0.55 & C05659 \\
\hline Lactulose 1 & Lactulose & $\mathrm{C} 12 \mathrm{H} 22 \mathrm{O} 11$ & -0.52 & C07064 \\
\hline 1-Monopalmitin & 1-Monopalmitin & ND & -0.22 & ND \\
\hline Glycolic acid & Glycolic acid & $\mathrm{C} 2 \mathrm{H} 4 \mathrm{O} 3$ & 0.4 & C00160 \\
\hline 11-Beta-prostaglandin-F-2-alpha 1 & 11-epi-Prostaglandin F2alpha & $\mathrm{C} 2 \mathrm{OH} 34 \mathrm{O} 5$ & 0.42 & C05959 \\
\hline 2-Hydroxy-3-isopropylbutanedioic acid & 2-Hydroxy-3-isopropylbutanedioic acid & ND & 0.45 & ND \\
\hline Phenylpyruvate & Phenylpyruvate & $\mathrm{C} 9 \mathrm{H} 8 \mathrm{O} 3$ & 0.5765 & C00166 \\
\hline 5'-Methylthioadenosine 1 & 5'-Methylthioadenosine & $\mathrm{C} 11 \mathrm{H} 15 \mathrm{~N} 5 \mathrm{O} 3 \mathrm{~S}$ & 0.79 & C00170 \\
\hline 4-Hydroxyphenylacetic acid & 4-Hydroxyphenylacetic acid & $\mathrm{C} 8 \mathrm{H} 8 \mathrm{O} 3$ & 1.009 & C00642 \\
\hline N-Epsilon-Acetyl-L-lysine 1 & N6-Acetyl-L-lysine & $\mathrm{C} 8 \mathrm{H} 16 \mathrm{~N} 2 \mathrm{O} 3$ & 1.728 & C02727 \\
\hline Glucoheptonic acid 1 & Glucoheptonic acid & & 2.03 & \\
\hline Oxamic acid & Oxamic acid & $\mathrm{C} 2 \mathrm{H} 3 \mathrm{NO} 3$ & 2.58 & C01444 \\
\hline Benzoylformic acid 3 & Benzoylformic acid & $\mathrm{C} 8 \mathrm{H} 6 \mathrm{O} 3$ & 3.09 & C02137 \\
\hline Neohesperidin & Neohesperidin & $\mathrm{C} 28 \mathrm{H} 34015$ & 18.826 & C09806 \\
\hline
\end{tabular}

the a-hydroxy acid family of carboxylic acids. It is used in various skin-care products. Biological production of glycolic acid requires glyoxylic acid circle. As intermediates, oxaloacetic acid and glyoxylic acid are aminated to generate aspartic acid and glycine, respectively.

\section{Diagram of a proposed OppA regulatory pathway for surfactin biosynthesis}

In terms of signal transduction, OppA has two possible modes of action: one is the binding of non-specific oligopeptide signal molecules, transporting them into the cell, activating the corresponding transcriptional regulatory factors by changing the intracellular amino acid concentration, and directing the downstream cellular metabolic activity; the second is the combination of specific oligopeptide signal molecules secreted by the bacteria to the outside, transmitting the signal into the cell, acting on the expression of the target gene, regulating cellular metabolic activities such as spore formation, splicing and pathogenicity [44-46]. According to the findings from this study, we proposed the hypothesis that mutations in the $O p p A$ gene may lead to the changes in oligopeptides acquisition. In other words, the mutated OppA protein may combine different types or different amounts of oligopeptides. Then the changed oligopeptides may activate or suppress the global regulator protein CcpA in CCR pathway. ComA and DegU may indirectly regulate surfactin synthesis in Bs-H74 mutant strain (Fig. 3).

Bacillus subtilis has been intensively studied for biological control of plant diseases. Previously, a mutant strain Bs-H74 demonstrated a 10\% stronger inhibitory activity against $R$. solani, the causal pathogen of rice sheath blight than the wild type Bs-916. HPLC analysis showed that Bs-H74 produces significantly more surfactin, a major antimicrobial substance. The $s r f A$ locus and ComA are required for the production of surfactin, but our data showed that the PsrfA and $\operatorname{com} A$ sequences were $100 \%$ conserved between the Bs-H74 and Bs-916 strains. These results suggested that promotion in surfactin production in the mutant may be due to other regulatory mechanisms.

To explore the underlying molecular mechanisms of the higher surfactin production in the mutant, highthroughput proteomic analysis was carried out to compare the protein expression between Bs-916 and Bs-H74. Proteome data showed that OppA, DegU and proteins in Carbon Catabolite Repression (CCR) may be involved in surfactin production. In order to test this hypothesis, we verified the transcription of the opp system genes. Data showed that both the oppA and oppF genes were upregulated in the Bs-H74 strain. The expression levels of CCR-associated genes are different between the wild type Bs-916 and the mutant Bs-H74. The central regulated gene of $\mathrm{CCR}, c c p A$ gene was upregulated in the Bs-H74 strain as we predicted. These results showed that oppA and $c c p A$ may be involved in surfactin production regulatory pathways. Through the SPINE (Strep-protein interaction experiment) method, 44 functionally known proteins that may interact with the CcpA protein were identified. Among these proteins, 7 proteins appear in our proteome data, which are RpoA, FtsZ, PurA, FbaA, Mdh, CitB, GlnA. In addition, SrfAA, SrfAB, SrfAC proteins are also included in these proteins. However, the authors did not further analyze the interaction between 


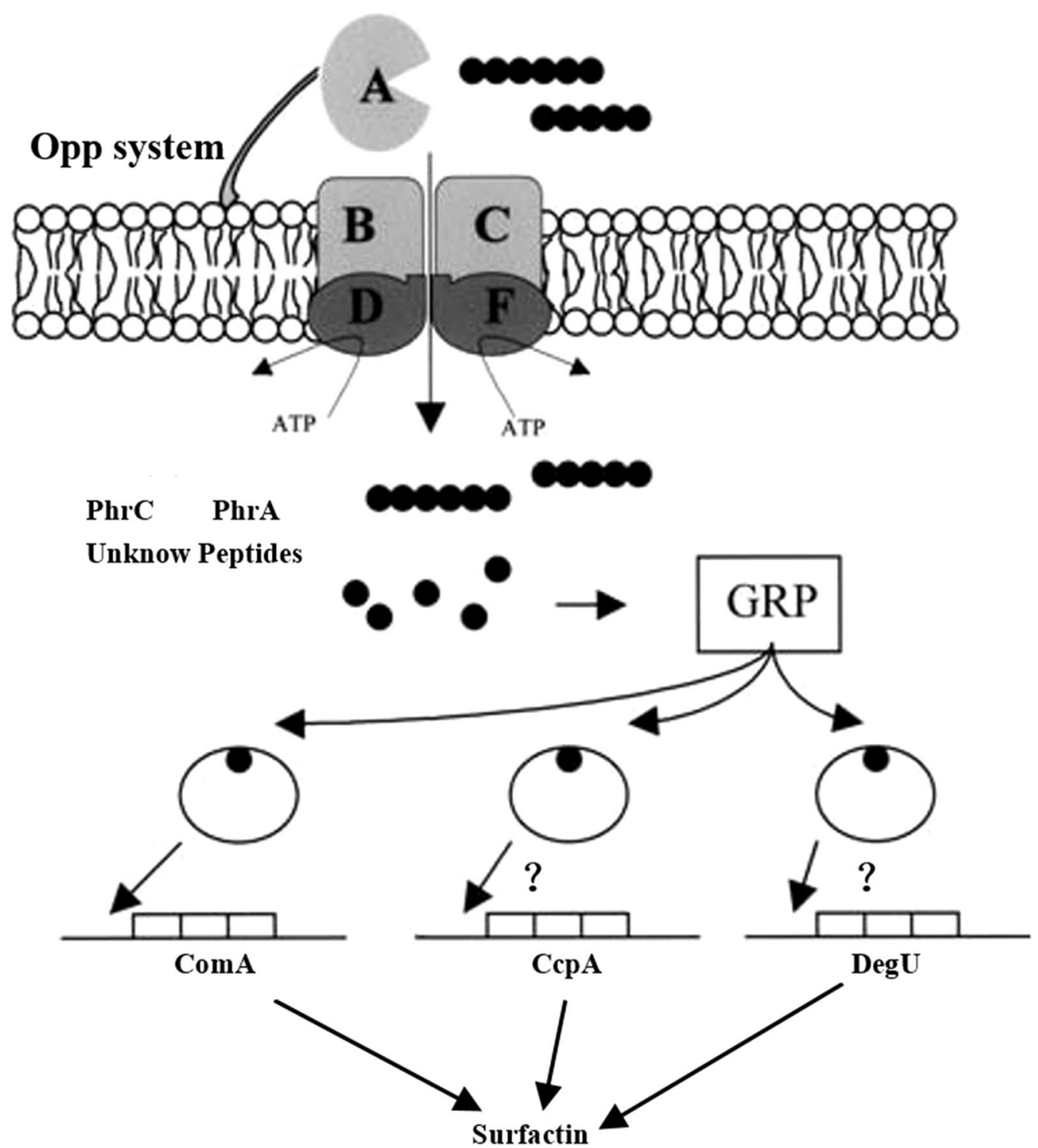

Fig. 3 Proposed diagram of Opp regulation of surfactin synthesis. Opp mediates the uptake of peptides, such as PhrA, PhrC and unknown peptides as a nutrient source. The acquisition of peptides indirectly influences comA, сcpA and $\operatorname{deg} U$ gene expression through an unknown mechanism, which may activate surfactin synthesis gene expression. GRP, global regulator protein, Black arrows, putative regulatory effects, Black arrows with question mark, unclear or indirect effects

CcpA protein and SrfAA, SrfAB, and SrfAC proteins. They provided the hypothesis that CcpA proteins interactive with RpoA and CodY by activating ackA (the gene encoding acetate kinase). Finally, RpoA and CodY proteins and CcpA can forms complexes, which regulate the upstream sequence of the ack $A$ promoter and activate gene transcription [47]. In this study, RpoA protein expression was up-regulated, consistent with the positive regulation of CcpA in the above studies. We also detected up-regulated expression of SrfAA, SrfAB, and SrfAC by RT-PCR, which implicated that CcpA may be positively regulate the expression of the srf operon. However, it is unclear that CcpA protein activates the synthesis of surfactin by directly binding to the srf operon, or indirectly by binding to the relevant factors of surfactin synthesis. Further studies on protein interaction will be carry out to clarify.

Transcriptional regulatory protein DegU is partner of the two-component regulatory system DegS/DegU which plays a critical role in the exponential growth period. DegS/DegU involved in the control of many genes expression of different biological processes, for example, 
biofilm formation, flagellum formation and competence for DNA uptake, positively or negatively regulates expression of many different genes. The phosphorylated form of $\mathrm{DegU}$ is important to synthesis of degradative enzymes, flagellum formation and biofilm formation. SrfA expression has been known to be activated by DegU-P, as the mutation of $\operatorname{deg} U$ causes downward expression of $s r f A$ gene in B. subtilis. DegU is also regulated indirectly by RghR. RghR represses Rap proteins by protein-protein interactions, which inhibit the binding of DegU to DNA, causing to inhibition of $\operatorname{srfA}$ expression $[48,49]$. In the present study, we found that the increased transcription/ expression of DegU was associated with the higher production of surfactin, which is consistent with previous studies.

And then we found a point mutation in the oppA gene in Bs-H74 through whole-genome resequencing. This SNP mutation is not located in "Venus's flytrap", suggesting that other regulatory mechanisms may exist. In previous studies on $C$. difficile, the opp operon was reported to be repressed by the important regulators CcpA, CodY and glucose, implying that the Opp transporter is involved in adjustment to supplement restricting conditions [50]. In B. subtilis, CcpA can bind directly to upstream region of the $\operatorname{deg} U$, implying that CcpA may be interacted with $\operatorname{deg} U$ by protein and DNA [51]. In this study, we detected that $c c p A$ and $\operatorname{deg} U$ genes were upregulated in the Bs-H74 strain implying that the two global regulatory proteins may be involved in oppA signaling pathway.

The biosynthesis of surfactin is catalysed through NRPS, initiated from the condensation of fatty acids and Glu. Other constituent amino acids, Asp, Leu, Val are assembled through the NRPS multi-enzyme complex, comprising adenylation, condensation, and thiolation domains responsible for the activation of amino acids and peptide chain elongation. The content of N6-acetylL-lysine and glycolic acid were found to be increased in Bs-H74. As intermediates, oxaloacetic acid and glyoxylic acid are aminated to generate Asp and Gly, respectively. In our study, MTA increased by 0.8 -fold. It is involved in the methionine salvage pathway. This pathway regulate some important metabolites, for example, $S$-adenosylmethionine (SAM). MTA is produced during polyamine synthesis, which is a suppressor of polyamine synthesis and transmethylation reaction. In the proteomic data of this study, the related proteins are detected, MetK, MetE and MtnD. Among them, MetK catalyze the reaction of methionine to form SAM, is up-regulated. So the increase of SAM content leads to an increase in MTA content. Shigeo Tojo et al. reported that the $m t h A$ gene point mutant was responsible for overproduction of bacilysin. This mutant increase in the SAM level was directly responsible for bacilysin overproduction, as confirmed by overexpression of the metK gene encoding SAM synthetase [52]. Based on these data, additional amino acids and SAM may be used to produce surfactin and provide a reasonable explanation. In this study, in addition to surfactin production increase, another unknown secondary metabolite, Neohesperidin-like flavonoid compound was synthesized in large quantities. In Bacillus, the KEGG pathway indicates that the synthesis of flavonoids involves phenylalanine metabolic pathway, which catabolite of pathway provide precursors for flavonoids synthesis. Metabolomics data showed that the related metabolite phenylpyruvate was increased increased by more than twofold. And SAM was frequently a methyl donor in the synthesis of flavonoids.

\section{Conclusion}

Subsequently, we proposed the hypothesis that the point mutation in the oppA gene may lead to changes in oligopeptides acquisition in B. subtilis, and then the changed oligopeptides may activate or suppress the global regulatory protein, CcpA in CCR pathway, and ComA and DegU may indirectly regulate surfactin synthesis in Bs-H74 mutant strain.

In our study, one residue has been mutated to a substitution, Trp for Arg in OppA protein. This SNP, W519R (TGG $\rightarrow$ CGG), is not a previously reported active site, which may be the key amino acid site that can change the regulating process. At both the transcriptional and translational levels, we suggested that OppA plays a key role in surfactin synthesis regulation. To further explore the regulatory mechanism in $\mathrm{Bs}-\mathrm{H} 74$, metabolomics studies were performed in this study. Interestingly, only 16 metabolites showed changes in abundance in Bs-H74 compared to Bs-916. It was found that metabolites related to amino acids metabolism, biosynthesis of secondary metabolites, biosynthesis of antibiotics, biosynthesis of amino acids, lipids and lipid-like molecules metabolism, and carbohydrates metabolic pathways were enhanced in the Bs-H74 mutant strain. Neohesperidin, a natural flavanone glycoside from citrus with a range of biological activities, increased by 18 times in Bs-H74 than the wild type Bs-916. The mutation in the oppA gene may have caused significant changes of many genes at transcriptional and translational levels, while only one or two metabolites have greatly increased their abundance. It suggested that the upstream regulation has enriched some certain metabolic pathways, thus increased the production of surfactin and a few other metabolites, such as Neohesperidin.

In summary, with the combination of the multi-omics data from this study and the information from previous research, we have identified the potential genes and 
regulators that could be positively or negatively regulating $\operatorname{srfA}$ expression. These findings have revealed the mechanism underlying the improved antagonistic property with increased surfactin production in Bs- $\mathrm{H} 74$. This study has provided a solid physical and theoretical basis for practically applying metabolic and genetic engineering to achieve improved and high-yielding biocontrol strains.

\section{Materials and methods}

\section{Strain and culture media}

Bacillus subtilis strains Bs-916 and mutant Bs-H74 was obtained and maintained in our lab [39]. Luria-Bertani (LB) medium was made as previously described [53]. B. subtilis strains were grown at $28{ }^{\circ} \mathrm{C}$ in either YPGA medium or YPG liquid medium with an agitation rate of $130 \mathrm{rpm}$. A single colony from YPGA solid media was transferred into YPG liquid media and then incubated for $24 \mathrm{~h}$ at $28^{\circ} \mathrm{C}$ in a shaker.

\section{Protein extraction}

Protein extractions were performed according to Faurobert et al. with minor modification [54]. Samples were ground to fine powder with liquid nitrogen, then suspended in 3 volumes of extraction buffer $(700 \mathrm{mM}$ sucrose, $500 \mathrm{mM}$ Tris, $\mathrm{pH}$ 8.0, $100 \mathrm{mM} \mathrm{KCl,} \%$ (v/v) $\beta$-mercaptoethanol, $2 \mathrm{mM}$ phenylmethylsulfonyl fluoride, $\mathrm{pH}$ 8.0) and incubated for $20 \mathrm{~min}$ on ice. Afterward, an equal volume of Tris-saturated phenol was added. Samples were shaken on ice for $20 \mathrm{~min}$ and then centrifuged (15 min, 5000g, $4{ }^{\circ} \mathrm{C}$ ). The phenolic phase was recovered and re-extracted with the same volume of extraction buffer. Subsequently, centrifugation was repeated and 4 volumes of precipitation solution $(0.1 \mathrm{M}$ ammonium acetate in methanol) were added to the recovered phenol phase. Protein was precipitated at $-20{ }^{\circ} \mathrm{C}$ overnight. After centrifugation $\left(15 \mathrm{~min}, 5000 \mathrm{~g}, 4^{\circ} \mathrm{C}\right)$, the protein pellet was washed twice with the precipitation solution and once with cold acetone. Protein pellet was lyophilized by vacuum, and stored at $-80{ }^{\circ} \mathrm{C}$ until use. Before 2-DE, protein pellets were dissolved in a lysis buffer (8 M urea, $2 \mathrm{M}$ thiourea, 4\% CHAPS, $13 \mathrm{mM}$ DTT, 2\% pharmalyte $3-10$ ) and used immediately after debris was removed by centrifugation as described above. The protein concentration was determined with Bio-Rad protein assay kit based on the Bradford method using BSA as standard. Three independent protein extractions were performed.

\section{2-DE and image analysis}

Samples were applied to $17 \mathrm{~cm}$ liner IPG strips $(\mathrm{pH}$ 4-7). After 14-h rehydration, isoelectric focusing was performed on PROTEAN IEF Cell (Bio-Rad) as following program: $30 \mathrm{~min}$ at $250 \mathrm{~V}, 1 \mathrm{~h}$ at $1000 \mathrm{~V}, 5 \mathrm{~h}$ to increase the voltage from 1000 to $8000 \mathrm{~V}, 7.5 \mathrm{~h}$ at $8000 \mathrm{~V}$. Gels were then subjected to $2 \times 20$ min equilibration, using buffer I and buffer II, which basically contains $6 \mathrm{M}$ Urea, $50 \mathrm{mM}$ Tris- $\mathrm{HCl}(\mathrm{pH} 8.8), 30 \%$ glycerol, $2 \%(\mathrm{w} / \mathrm{v})$ SDS, with additional $2 \%(\mathrm{w} / \mathrm{v})$ DTT (I) and $2.5 \%(\mathrm{w} / \mathrm{v})$ iodoacetamide(II) respectively. SDS-PAGE was performed with $12 \%$ acrylamide gels in the PROTEAN MINI 2 (Bio-Rad) for $5 \mathrm{~h}$ at $180 \mathrm{~V}$. PI and molecular mass of the protein were determined respectively by the liner $\mathrm{pH}$ arrangement of IPG strips and SDS-PAGE marker. Proteins were visualized with Coomassie Brilliant Blue (CBB) G250. Protein spots were detected by PDQuest software (Bio-Rad) and normalized to total quantity in valid spots under PPM $(\times 1,000,000)$ scaling step. According to the PDQuest software, spot quantity is the total intensity of a defined spot in a gel image. The intensity is the sum of intensities of the image pixels inside a boundary. It is calculated during spot detection and Gaussian fitting, which is calculated by PDQuest software. After the manual process, the well-separated spots in all triplicate gels were analyzed by Students' T test $(\mathrm{P}<0.05)$.

\section{Tryptic digestion and protein identification}

Protein spots were excised from gels, destained for $20 \mathrm{~min}$ in $30 \mathrm{mM}$ potassium ferricyanide/100 mM sodium thiosulfate $(1: 1 \mathrm{v} / \mathrm{v})$ and washed in Milli-Q water until the gels were destained. The spots were kept in $0.2 \mathrm{M} \mathrm{NH}_{4} \mathrm{HCO}_{3}$ for $20 \mathrm{~min}$ and then lyophilizated. Each spot was digested overnight in $2 \mu \mathrm{L} 12.5 \mathrm{ng} / \mu \mathrm{L}$ trypsin in $0.1 \mathrm{M} \mathrm{NH}_{4} \mathrm{HCO}_{3}$. The peptides were extracted with $50 \%$ ACN, 0.1\% TFA three times. All mass spectra were acquired on an AutoFlex MALDI-TOF/TOF mass spectrometer with LIFT technology (Bruker Daltonics, Bremen, Germany). Tryptic digests were prepared on AnchorChip sample plate (Bruker Daltonics, Bremen, Germany) according to the manufacturer's instructions. $\mathrm{MS} / \mathrm{MS}$ data were acquired with a $\mathrm{N}_{2}$ laser at $25-\mathrm{Hz}$ sampling rate. The data set was submitted to MASCOT for protein identification. National Center for Biotechnology non-redundant (NCBInr 20080221) protein database (6122577 sequences; 2096230148 residues) was searched against. The search was performed using green plants as taxonomy, which contained 473596 sequences. Other parameters for searching were enzyme of trypsin, one missed cleavage, fixed modifications of carbamidomethyl (C), and variable modifications of oxidation (Met). Peptide tolerance of $100 \mathrm{ppm}$, fragment mass tolerance of $\pm 0.5 \mathrm{Da}$, and peptide charge of $1+$ were selected. Only significant hits, as defined by the MASCOT probability analysis $(\mathrm{P}<0.05)$, were accepted. 


\section{Functional annotation of proteins}

Overrepresentation of gene ontology (GO) terms for differentially abundant proteins was calculated with the Biological Network Gene Ontology Tool (BiNGO v. 2.44 [55]) and visualized in Cytoscape [56]. The hypergeometric test used for the enrichment analysis in BiNGO was performed using a FDR-corrected P-value cutoff of 0.05 and using all GO annotated genes of Bs168 as a background reference. The annotation was done using the Basic Local Alignment Search Tool for proteins (BLASTp) with default settings.

\section{Real-time PCR}

Quantitative real-time RT-PCR analysis was performed by ABI Stepone plus using SybrGreen (Sangon Ltd) technology on ABI Stepone plus (Applied Biosystems, Foster City, USA). RNA was extracted by RNAprep pure kit (TIANGEN Ltd, Beijing, China). One to two microliters of RNA solution was digested with DNase I (TaKaRa Biotech. Co. Ltd, Dalian, China) and used in the RT-PCR analyses. RT was initiated in the presence of oligo-(dT) primers $\left(42{ }^{\circ} \mathrm{C}, 30 \mathrm{~min}\right)$, and, after inactivation of the reverse transcriptase $\left(95{ }^{\circ} \mathrm{C}, 5 \mathrm{~min}\right)$, the appropriate primers (Table 4) were added for PCR cycling (3 min at $95{ }^{\circ} \mathrm{C}, 40$ cycles of $7 \mathrm{~s}$ at $95^{\circ} \mathrm{C}, 10 \mathrm{~s}$ at $57^{\circ} \mathrm{C}$, and $15 \mathrm{~s}$ at $72{ }^{\circ} \mathrm{C}$ ). Amplification of a constitutively expressed gene (16sRNA) served as an internal control in the RT-PCR assays.

\section{Resequencing}

Genomic DNA $(5 \mu \mathrm{g})$ isolated from single colonies of the endpoint strains was used to generate the genomic DNA library using the Illumina genomic DNA library generation kit following the manufacturer's protocol (Illumina Inc., San Diego, CA, USA). Briefly, bacterial genomic DNA was fragmented by nebulization. The ends of fragmented DNA were repaired by T4 DNA polymerase, Klenow DNA polymerase, and T4 polynucleotide kinase. The Klenow exo minus enzyme was then used to add an 'A' base to the 3 ' end of the DNA fragments. After the ligation of the adapters to the ends of the DNA fragments, the ligated DNA fragments were subjected to $2 \% 1 \times$ TAE agarose gel electrophoresis. DNA fragments ranging from 150 to $300 \mathrm{bp}$ were recovered from the gel and purified using the Qiagen mini gel purification kit. Finally, the adapter-modified DNA fragments were enriched by PCR. The final concentration of the genomic DNA library was determined by Nano drop and validated by running $2 \% 1 \times \mathrm{TAE}$ agarose gel electrophoresis. A $4 \mathrm{pM}$ genomic DNA library was used to generate the cluster on the Flowcell following the manufacturer's protocol. The genomic sequencing primer v2 was used for all DNA sequencing. A 36 cycle sequencing run was carried

\begin{tabular}{|c|c|}
\hline Oligonucleotide & Oligonucleotide sequences \\
\hline $\operatorname{com} A$ & $\begin{array}{l}5^{\prime} \text { ATGAAAAAGATACTAGTGATTGATG } 3^{\prime} \\
5^{\prime} \text { TTAAAGTACACCGTCTGATTTC } 3^{\prime}\end{array}$ \\
\hline Psrfa & $\begin{array}{l}\text { 5' AAAATGTCATGAAAGAATCGTTGTAAG 3' } \\
\text { 5' CGCAAGATTTGAAATGCTCGTGTGA } 3^{\prime}\end{array}$ \\
\hline $\operatorname{ccp} A$ & $\begin{array}{l}5^{\prime} \text { CAAAAGTCTCATCGCCACG 3' } \\
\text { 5' CGAGATTGAGCCTGATGGTC } 33^{\prime}\end{array}$ \\
\hline $\operatorname{cgg} R$ & $\begin{array}{l}5^{\prime} \text { ATCAGGCTTACCGAACCGA } 3^{\prime} \\
5^{\prime} \text { GCGTCAACCCTAAAACATCC } 3^{\prime}\end{array}$ \\
\hline citB & $\begin{array}{l}\text { 5' ACCGTTGTTGCGGCAGTAT 3' } \\
5^{\prime} \text { TCCTGGTGTTGCTGAGCTTC } 3^{\prime}\end{array}$ \\
\hline gapA & $\begin{array}{l}\text { 5' CATTCCTACATCAACTGGTGCTG } 3^{\prime} \\
\text { 5' CATTCCTACATCAACTGGTGCTG } 3^{\prime}\end{array}$ \\
\hline$f b p$ & $\begin{array}{l}\text { 5' ATAACGGCAACCTGCTGATT 3' } \\
5^{\prime} \text { GTATTCGCCTGTCCATAAGTACC } 3^{\prime}\end{array}$ \\
\hline 16sRNA & $\begin{array}{l}5^{\prime} \text { GAGGCAGCAGTAGGGAATCTT } 3^{\prime} \\
5^{\prime} \text { CCGTGGCTTTCTGGTTAGGT 3' }\end{array}$ \\
\hline
\end{tabular}

out using the Illumina analyzer following the manufacturer's protocol.

The whole-genome sequencing and mutation detection Bs-H74 mutant strain was sequenced on Illumina GA, GA II, and GA IIx instruments. The resulting reads were obtained. Reads were mapped to the reference genome of the Bs-916 strain (GenBank accession no. AFSU00000000 [57]), and mutations were predicted using the breseq computational pipeline [58]. This pipeline detects point mutations, deletions, and new sequence junctions that may indicate IS-element insertions or other rearrangements, as described in its online documentation. Large duplications and amplifications were predicted manually by examining the depth of read coverage across each genome.

\section{Sample preparation for metabolite identification}

Samples were taken as quickly as possible from the fermentor and immediately quenched to halt cellular metabolism, at $-45^{\circ} \mathrm{C}$ in $0.4 \mathrm{~mL}$ methanol extraction liquid (V methanol: V chloroform=3:1). $20 \mu \mathrm{L}$ of L-2-Chlorophenylalanine was added as an internal standard to the sample. The intracellular metabolites were extracted from the cell suspension with a ball mill. Samples were homogenized in a ball mill for $4 \mathrm{~min}$ at $45 \mathrm{~Hz}$, ultrasound treated for $5 \mathrm{~min}$ (incubated in ice water) for 5 times, and then centrifuged for $15 \mathrm{~min}$ at $12,000 \mathrm{rpm}$ at $4{ }^{\circ} \mathrm{C}$. The supernatant was transferred $(0.39 \mathrm{~mL})$ into a fresh $2 \mathrm{ml}$ GC/MS glass vial. $15 \mu \mathrm{L}$ of supernatant from each sample was pooled as QC sample. Cell extracts were dried in a vacuum concentrator without heating. The dried 
extracts were derivatized with $20 \mu \mathrm{L}$ of methoxy amination hydrochloride $(20 \mathrm{mg} / \mathrm{mL}$ in pyridine) for $30 \mathrm{~min}$ at $80{ }^{\circ} \mathrm{C}$. Subsequently, the extracts were silylated for $2 \mathrm{~h}$ at $70{ }^{\circ} \mathrm{C}$ with $30 \mu \mathrm{L}$ of the BSTFA regent (1\% TMCS, v/v). $10 \mu \mathrm{L}$ of FAMEs (Standard mixture of fatty acid methyl esters, C8-C16:1 mg/mL; C18-C24:0.5 mg/mL in chloroform) was added to the QC sample. After samples cooled down to the room temperature, they were mixed thoroughly for GC-TOFMS analysis.

\section{GC-TOFMS analyses for metabolite identification}

GC-TOFMS analysis was performed using an Agilent 7890 gas chromatograph system coupled with a Pegasus HT time-of-flight mass spectrometer. The system utilized a DB-5MS capillary column coated with 5\% diphenyl cross-linked with 95\% dimethylpolysiloxane (30 $\mathrm{m} \times 250 \mu \mathrm{m}$ inner diameter, $0.25 \mu \mathrm{m}$ film thickness; J\&W Scientific, Folsom, CA, USA). A $1 \mu \mathrm{L}$ aliquot of the analyte was injected in splitless mode. Helium was used as the carrier gas, the front inlet purge flow was $3 \mathrm{~mL} /$ min, and the gas flow rate through the column was $1 \mathrm{~mL} /$ min. The initial temperature was kept at $50{ }^{\circ} \mathrm{C}$ for $1 \mathrm{~min}$, then raised to $310{ }^{\circ} \mathrm{C}$ at a rate of $20{ }^{\circ} \mathrm{C} / \mathrm{min}$, then kept for $5 \mathrm{~min}$ at $310^{\circ} \mathrm{C}$. The injection, transfer line, and ion source temperatures were 280,270 , and $220{ }^{\circ} \mathrm{C}$, respectively. The energy was $-70 \mathrm{eV}$ in electron impact mode. The mass spectrometry data were acquired in full-scan mode with the $\mathrm{m} / \mathrm{z}$ range of $50-500$ at a rate of 20 spectra per second after a solvent delay of $455 \mathrm{~s}$.

Chroma TOF 4.3X software of LECO Corporation and LECO-Fiehn Rtx 5 database were used for raw peaks exacting, the data baselines filtering and calibration of the baseline, peak alignment, deconvolution analysis, peak identification and integration of the peak area. The RI (retention time index) method was used in the peak identification, and the RI tolerance was 5000. Remove metabolic features detected in $<50 \%$ of QC samples.

\section{Additional file}

Additional file 1: Figure S1. Opp system in Bacillus, oligopeptide ABC transporter family. A, an extracellular ligand-binding lipoprotein (OppA), two transmembrane proteins (OppBC) that form a membrane-spanning pore, and two cytoplasmic ATPases (OppDF) that drive the transport of the peptide into the cell; B, 3D structure of OppA protein; C, Identification of oligopeptide transporter systems in Bs-916.

\section{Authors' contributions}

$X W$ designed, performed the majority of the experiment work, analyzed data, and wrote the manuscript. HF and XC helped in analyzing data. ZC and LW were responsible for the original concept and supervised the work. All authors read and approved the final manuscript.

\section{Funding}

This work was supported by a Special Fund for Agro-scientific Research in the Public Interest (Grant No. 201403032), National Natural Science Foundation of China (Grant No. 31601620), Natural Science Foundation of JiangSu Province of China (Grant No. BK2012782).

\section{Ethics approval and consent to participate}

Not applicable.

\section{Consent for publication}

Not applicable.

\section{Competing interests}

The authors declare that they have no competing interests.

Received: 24 February 2019 Accepted: 30 July 2019

Published online: 19 August 2019

\section{References}

1. Kloepper JW, Ryu CM, Zhang S. Induced systemic resistance and promotion of plant growth by Bacillus spp. Phytopathology. 2004;94:1259-66.

2. Arima K, Kakinuma A, Tamura G. Surfactin, a crystalline peptidelipid surfactant produced by Bacillus subtilis: isolation, characterization and its inhibition of fibrin clot formation. Biochem Biophys Res Commun. 1968;31:488-94.

3. Hosono K, Suzuki H. Acylpeptides, the inhibitors of cyclic adenosine 3',5'-monophosphate phosphodiesterase. I. Purification, physicochemical properties and structures of fatty acid residues. J Antibiot (Tokyo). 1983;36:667-73.

4. Kakinuma A, Hori M, Isono M, Tamura G, Arima K. Determination of amino acid sequence in surfactin, a crystalline peptide lipid surfactant produced by Bacillus subtilis. Agric Biol Chem. 1969;33:971-2.

5. Besson F, Peypoux F, Michel G, Delcambe L. Identification of antibiotics of iturin group in various strains of Bacillus subtilis. J Antibiot (Tokyo). 1978;31:284-8.

6. Vanittanakom N, Loeffler W, Koch U, Jung G. Fengycin, a novel antifungal lipopeptide antibiotic produced by Bacillus subtilis F-29-3. J Antibiot (Tokyo). 1986;39:888-901.

7. Reuter K, Mofid MR, Marahiel MA, Ficner R. Crystal structure of the surfactin synthetase-activating enzyme Sfp: a prototype of the 4'-phosphopantetheinyl transferase superfamily. EMBO J. 1999;18:6823-31.

8. Nakano MM, Marahiel MA, Zuber P. Identification of a genetic locus required for biosynthesis of the lipopeptide antibiotic surfactin in Bacillus subtilis. J Bacteriol. 1988;170:5662-8.

9. Nakano MM, Magnuson R, Myers A, Curry J, Grossman AD, Zuber P. srfA is an operon required for surfactin production, competence development, and efficient sporulation in Bacillus subtilis. J Bacteriol. 1991;173:1770-8.

10. Guillen N, Weinrauch Y, Dubnau DA. Cloning and characterization of the regulatory Bacillus subtilis competence genes comA and comB. J Bacteriol. 1989;17:5354-61.

11. Bourret RB, Hess JF, Borkovich KA, Pakula AA, Simon MI. Protein phosphorylation in chemotaxis and two-component regulatory systems of bacteria. J Biol Chem. 1989;264:7085-8.

12. Weinrauch Y, Penchev R, Dubnau E, Smith I, Dubnau DA. Bacillus subtilis regulatory gene product for genetic competence and sporulation resembles sensor protein members of the bacterial two-component signal-transduction systems. Genes Dev. 1990;4:860-72.

13. Ohno A, Ano T, Shoda M. Production of a lipopeptide antibiotic, surfactin, by recombinant Bacillus subtilis in solid state fermentation. Biotechnol Bioeng. 1995:47:209-14.

14. Wei $Y$, Chu I. $\mathrm{Mn}^{2+}$ improves surfactin production by Bacillus subtilis. Biotechnol Lett. 2002;24:479-82.

15. Wei YH, Wang LF, Chang JS. Optimizing iron supplement strategies for enhanced surfactin production with Bacillus subtilis. Biotechnol Prog. 2004;20:979-83.

16. Abdel-Mawgoud AM, Aboulwafa MM, Hassouna NA. Characterization of surfactin produced by Bacillus subtilis isolate BS5. Appl Biochem Biotechnol. 2008;150:289-303. 
17. Mukherjee S, Das P, Sen R. Towards commercial production of microbial surfactants. Trends Biotechnol. 2006;24:509-15.

18. Jung J, Yu KO, Ramzi AB, Choe SH, Kim SW, Han SO. Improvement of surfactin production in Bacillus subtilis using synthetic wastewater by overexpression of specific extracellular signaling peptides, comX and phrC. Biotechnol Bioeng. 2012;109:2349-56.

19. Willenbacher J, Mohr T, Henkel M, Gebhard S, Mascher T, Syldatk C, et al. Substitution of the native srfA promoter by constitutive Pveg in two $B$. subtilis strains and evaluation of the effect on Surfactin production. $J$ Biotechnol. 2016;224:14-7.

20. Wang M, Yu H, Shen Z. Antisense RNA-based strategy for enhancing surfactin production in Bacillus subtilis TS1726 via overexpression of the unconventional biotin Carboxylase II to enhance ACCase activity. ACS Synth Biol. 2019;15:251-6.

21. Wu Q, Zhi Y, Xu Y. Systematically engineering the biosynthesis of a green biosurfactant surfactin by Bacillus subtilis 168. Metab Eng. 2019;52:87-97.

22. Wang $Q, Y u H$, Wang $M$, Yang $H$, Shen Z. Enhanced biosynthesis and characterization of surfactin isoforms with engineered Bacillus subtilis through promoter replacement and Vitreoscilla hemoglobin co-expression. Process Biochem. 2018;70:36-44.

23. Tynkkynen S, Buist G, Kunji E, Kok J, Poolman B, Venema G, et al. Genetic and biochemical characterization of the oligopeptide transport system of Lactococcus lactis. J Bacteriol. 1993;175:7523-32.

24. Doeven MK, Abele R, Tampe R, Poolman B. The binding specificity of OppA determines the selectivity of the oligopeptide ATP-binding cassette transporter. J Biol Chem. 2004;279:32301-7.

25. Monnet V. Bacterial oligopeptide-binding proteins. Cell Mol Life Sci. 2004;60:2100-14.

26. Mao B, Pear MR, McCammon JA, Quiocho FA. Hinge-bending in L-arabinose-binding protein. The "Venus's-flytrap" model. J Biol Chem. 1982;257:1131-3.

27. Zheng L, Chen Z, Itzek A, Herzberg MC, Kreth J. CcpA regulates biofilm formation and competence in Streptococcus gordonii. Mol Oral Microbiol. 2012;27:83-94

28. Galinier A, Kravanja M, Engelmann R, Hengstenberg W, Kilhoffer MC, Deutscher J, et al. New protein kinase and protein phosphatase families mediate signal transduction in bacterial catabolite repression. Proc Natl Acad Sci USA. 1998;95:1823-8

29. Weinrauch Y, Guillen N, Dubnau DA. Sequence and transcription mapping of Bacillus subtilis competence genes comB and comA, one of which is related to a family of bacterial regulatory determinants. J Bacteriol. 1989;171:5362-75.

30. Reizer J, Hoischen C, Titgemeyer F, Rivolta C, Rabus R, Stulke J, et al. A novel protein kinase that controls carbon catabolite repression in bacteria. Mol Microbiol. 1998;27:1157-69.

31. Jault JM, Fieulaine S, Nessler S, Gonzalo P, Di Pietro A, Deutscher J, et al. The HPr kinase from Bacillus subtilis is a homo-oligomeric enzyme which exhibits strong positive cooperativity for nucleotide and fructose 1,6-bisphosphate binding. J Biol Chem. 2000;275:1773-80.

32. Ramstrom H, Sanglier S, Leize-Wagner E, Philippe C, Van Dorsselaer A, Haiech J. Properties and regulation of the bifunctional enzyme HPr kinase/phosphatase in Bacillus subtilis. J Biol Chem. 2003;278:1174-85.

33. Kundig W, Ghosh S, Roseman S. Phosphate bound to histidine in a protein as an intermediate in a novel phospho-transferase system. Proc Natl Acad Sci USA. 1964;52:1067-74.

34. Deutscher J, Herro R, Bourand A, Mijakovic I, Poncet S. P-Ser-HPr-a link between carbon metabolism and the virulence of some pathogenic bacteria. Biochim Biophys Acta. 2005;1754:118-25.

35. Gorke B, Stulke J. Carbon catabolite repression in bacteria: many ways to make the most out of nutrients. Nat Rev Microbiol. 2008;6:613-24.

36. Kim HJ, Roux A, Sonenshein AL. Direct and indirect roles of CcpA in regulation of Bacillus subtilis Krebs cycle genes. Mol Microbiol. 2002;45:179-90.

37. Kim HJ, Jourlin-Castelli C, Kim SI, Sonenshein AL. Regulation of the bacillus subtilis ccpC gene by $c c p A$ and $c c p C$. Mol Microbiol. 2002;43:399-410.

38. Chen Z, Xu Z. Evaluation and utilization of antagonistic bacteria against rice sheath blight. Chin J Rice Sci. 2000;14:98-102.
39. Li D, Nie F, Wei L, Wei B, Chen Z. Screening of high-yielding biocontrol bacterium Bs-916 mutant by ion implantation. Appl Microbiol Biotechnol. 2007;75:1401-8.

40. Zhang J, Sun C, Yan Y, Chen Q, Luo F, Zhu X, et al. Purification of naringin and neohesperidin from Huyou (Citrus changshanensis) fruit and their effects on glucose consumption in human HepG2 cells. Food Chem. 2012;135:1471-8

41. Hwang SL, Yen GC. Neuroprotective effects of the citrus flavanones against $\mathrm{H}_{2} \mathrm{O}_{2}$-induced cytotoxicity in PC12 cells. J Agric Food Chem. 2008;56:859-64.

42. Bellocco E, Barreca D, Laganà G, Leuzzi U, Tellone E, Ficarra S, et al. Influence of L-rhamnosyl-D-glucosyl derivatives on properties and biological interaction of flavonoids. Mol Cell Biochem. 2009;321:165-71.

43. Tan Z, Cheng J, Liu Q, Zhou L, Kenny J, Wang T, et al. Neohesperidin suppresses osteoclast differentiation, bone resorption and ovariectomisedinduced osteoporosis in mice. Mol Cell Endocrinol. 2017:439:369-78.

44. Magnuson R, Solomon J, Grossman AD. Biochemical and genetic characterization of a competence pheromone from B. subtilis. Cell. 1994;77:207-16.

45. Pearce BJ, Naughton AM, Masure HR. Peptide permeases modulate transformation in Streptococcus pneumoniae. Mol Microbiol. 1994;12:881-92.

46. Solomon JM, Lazazzera BA, Grossman AD. Purification and characterization of an extracellular peptide factor that affects two different developmental pathways in Bacillus subtilis. Genes Dev. 1996;10:2014-24.

47. Wünsche A, Hammer E, Bartholomae M, Völker U, Burkovski A, Seidel G, et al. CcpA forms complexes with CodY and RpoA in Bacillus subtilis. FEBS J. 2012;279:2201-14.

48. Hayashi K, Ohsawa T, Kobayashi K, Ogasawara N, Ogura M. The $\mathrm{H}_{2} \mathrm{O}_{2}$ stress-responsive regulator PerR positively regulates srfA expression in Bacillus subtilis. J Bacteriol. 2005;187:6659-67.

49. Hayashi K, Kensuke T, Kobayashi K, Ogasawara N, Ogura M. Bacillus subtilis RghR (YvaN) represses rapG and rapH, which encode inhibitors of expression of the srfA operon. Mol Microbiol. 2006;59:1714-29.

50. Edwards AN, Nawrocki KL, McBride SM. Conserved oligopeptide permeases modulate sporulation initiation in Clostridium difficile. Infect Immun. 2014;82:4276-91.

51. Ishii H, Tanaka T, Ogura M. The Bacillus subtilis response regulator gene deg $U$ is positively regulated by $C c p A$ and by catabolite-repressed synthesis of ClpC. J Bacteriol. 2014;195:193-201.

52. Tojo S, Kim JY, Tanaka Y, Inaoka T, Hiraga Y, Ochi K. The mthA mutation conferring low-level resistance to streptomycin enhances antibiotic production in Bacillus subtilis by increasing the S-adenosylmethionine pool size. J Bacteriol. 2014;196:1514-24

53. Sambrook J. Molecular cloning: a laboratory manual. 3rd ed. New York: Cold Spring Harbor Laboratory Press; 2007.

54. Faurobert M, Pelpoir E, Chaib J. Phenol extraction of proteins for proteomic studies of recalcitrant plant tissues. Methods Mol Biol. 2007;355:9-14.

55. Maere S, Heymans K, Kuiper M. BiNGO: a Cytoscape plugin to assess overrepresentation of gene ontology categories in biological networks. Bioinformatics. 2007;21:3448-9.

56. Shannon P, Markiel A, Ozier O, Baliga NS, Wang JT, Ramage D, et al. Cytoscape: a software environment for integrated models of biomolecular interaction networks. Genome Res. 2007;13:2498-504.

57. Wang X, Luo C, Chen Z. Genome sequence of the plant growth-promoting rhizobacterium Bacillus sp. strain 916. J Bacteriol. 2012;194:5467-8.

58. Blount ZD, Barrick JE, Davidson CJ, Lenski RE. Genomic analysis of a key innovation in an experimental Escherichia coli population. Nature. 2012;489:513-8.

\section{Publisher's Note}

Springer Nature remains neutral with regard to jurisdictional claims in published maps and institutional affiliations. 